\title{
Zur Sichtbarkeit und Verbreitung rechtswissenschaftlicher Dissertationen
}

Eric W. Steinhauer
A. Rechtswissenschaft und Open
Access
B. Geringe Rezeption von Disserta- tionen
C. Die Verbreitung Hagener juristi- scher Dissertationen in Biblio- theken
D. Die Verbreitung Frankfurter juristischer Dissertationen in Bibliotheken
E. Die Pflicht zur angemessenen Veröffentlichung
F. Die Dissertation als bibliotheka- rischer Ballast

G. Die elektronische Dissertation ...

H. Adressatenkreis von juristischen Dissertationen .................. 43

I. Wer soll das alles lesen? .......... 44

J. Eine freiwillige Angelegenheit? ........................... 45

K. Ein Wort zum Buch! ............. 48

L. Haben Verlage noch eine Zukunft?

M. Zusammenfassung und Ausblick

\section{A. Rechtswissenschaft und Open Access}

Die seit gut zwanzig Jahren intensiv geführte Debatte um Open Access hat in der Publikationspraxis der deutschen Rechtswissenschaft kaum Spuren hinterlassen. ${ }^{1}$ Immer noch gilt die bereits 2007 getroffene Feststellung von Christoph Engel und Wolfgang Schön, dass Juristen „ihre Text nicht im Internet zur Verfügung“ stellen. ${ }^{2}$ Diese Feststellung verwundert heute umso mehr, als die Arbeit mit digitalen Ressourcen mittlerweile ganz selbstverständlich zum juristischen Berufsalltag gehört. Man denke nur an das praktisch unverzichtbare Angebot von Beck-Online, ${ }^{3}$ das jedoch nur gegen Bezahlung zugänglich ist und damit einen wesentlichen Grundgedanken von Open Access nicht erfüllt, nämlich für Jedermann frei verfügbar zu sein. ${ }^{4}$ Offenbar hat die Digitalisierung des rechtswissenschaftlichen Arbeitens bislang nur die Form, nicht aber die überkommenen Publikationswege über juristische Verlage verändert. Das ist erstaunlich. Erfreut sich doch die Rechtswissenschaft wie kaum ein anderes Fach einer allgemeinen und digitalen Zugänglichkeit ihrer Quellen. Nach $\mathbb{} 5$ UrhG unterliegen Gesetze, Parlamentsmaterialien so-

1 Zur Open-Access-Bewegung allgemein Deppe/Beucke, Ursprünge und Entwicklung von Open Access, Söllner/Mittermaier, Praxishandbuch Open Access, Berlin [u.a.] 2017, S. 12-20; Krujatz, Open Access, Tübingen 2012, S. 40-57.

2 Vgl. Engel/Schön, Vorwort, in: Engel/Schön (Hrsg.), Das Proprium der Rechtswissenschaft, Tübingen 2007, S. [IX]. Für den Bereich der Zeitschriften wies die Rechtswissenschaft 2015 in internationaler Perspektive einen Open-Access-Anteil von weniger als 10\% auf, vgl. Herb, Sozialwissenschaften, in: Söllner/Mittermaier, Praxishandbuch Open Access, Berlin [u.a.] 2017, S. 255.

3 Dazu Beck, beck-online - die Datenbank - Publizieren in der digitalen Welt, in: Wesel/Beck (Hrsg.), 250 Jahre rechtswissenschaftlicher Verlag C. H. Beck : 1763 - 2013, München 2013, S. 521-533.

4 Dazu Fournier, Open-Access-Policies und ihre Gestaltung durch Forschungsförderer, in: Söllner/ Mittermaier, Praxishandbuch Open Access, Berlin [u.a.] 2017, S. 21. 
wie Gerichtsurteile als amtliche Werke keinem urheberrechtlichen Schutz. ${ }^{5}$ Sie sind, sieht man von gewissen Einschränkungen bei der Rechtsprechung ab, mittlerweile fast vollständig online und kostenfrei verfügbar. Durch die einfache Zugänglichkeit zu Gesetzesmaterialen lassen sich mit der gestiegenen Beliebtheit der historisch-genetischen Auslegung sogar schon methodische Konsequenzen des offenen Zugangs beobachten. ${ }^{6}$ Das scheinen auch die Verfasser solcher Materialien einzukalkulieren. Man hat den Eindruck, dass die Qualität von Gesetzesbegründungen, die durch ihre Publikation im Internet jetzt auf ein größeres Lesepublikum rechnen dürfen, in letzter Zeit spürbar angezogen hat. Mitunter erlauben sich die Referentinnen und Referenten eigene obiter dicta und manche ausholende Weitschweifigkeit, die der Gesetzesbegründung fast schon den Charakter eines Kommentars verleihen. ${ }^{7}$ Offenheit im Zugang führt nicht nur zu einer besseren Wahrnehmung. Sie scheint sich auch positiv auf die Qualität des Inhalts einer Publikation auszuwirken.

Dass juristische Autorinnen und Autoren auf diese Vorteile bislang verzichten, könnte zweierlei Ursachen haben. Zum einen wird die deutsche Publikationslandschaft in der Rechtswissenschaft von wenigen Akteuren dominiert, so dass eine Datenbank wie Beck-Online die Sichtbarkeit bei der professionellen Leserschaft und die Verfügbarkeit der Texte ausreichend und mit recht hohem Komfort sicherstellt. Zum anderen vergüten die juristischen Verlage gerade im Zeitschriftenbereich ihre Autorinnen und Autoren recht ordentlich, so dass es wenige Anreize gibt, die eingefahrenen Publikationsgewohnheiten zu ändern, auch wenn man selbst die freie Verfügbarkeit von Rechtsquellen im Netz durchaus schätzt. Die Enthaltsamkeit bei Open Access, die bei den juristischen Fachpublikationen, für die es in Justiz und Anwaltschaft einen durchaus lukrativen Markt gibt, zu beobachten ist, setzt sich auch bei den Dissertationen fort. Obwohl die Kultusministerkonferenz schon 1997 die so genannte elektronische Dissertation als regulären Veröffentlichungsweg anerkannt hat, ${ }^{8}$ ist die Zahl der online publizieren Doktorarbeiten im Fach Rechtswissenschaft im Vergleich zu Verlagsdissertationen immer noch

5 Vgl. Dreier, in: Dreier/Schulze, UrhG, 6. Aufl., München 2018, $\$$ 5, Rn. 5,6 und 11.

6 Vgl. Knauer, Juristische Methodenlehre 2.0? Der Wandel der juristischen Publikationsformate und sein Einfluss auf die juristische Methodenlehre, in: Rechtstheorie 40 (2009), S. 379-403.

7 Besonders markante Beispiele stellen etwa die Begründung zum Gesetzentwurf der Bundesregierung „Entwurf eines Gesetzes zur Angleichung des Urheberrechts an die aktuellen Erfordernisse der Wissensgesellschaft (Urheberrechts-Wissensgesellschafts-Gesetz - UrhWissG) “ BT-Drs. 18/12329 vom 15. Mai 2019 oder zum Gesetzentwurf der Landesregierung „Gesetz zur Förderung und Entwicklung der Kultur, der Kunst und der kulturellen Bildung in Nordrhein-Westfalen (Kulturfördergesetz NRW)“ LT-Drs. 16/6637 vom 27. August 2014 dar.

8 Grundsätze für die Veröffentlichung von Dissertationen - Beschluss der Kultusministerkonferenz vom 30. Oktober 1997, abgedruckt etwa in: Bibliotheksdienst 32 (1998), S. 750. 
verschwindend klein. ${ }^{9}$ Dies verwundert umso mehr, als Verlage für diese Publikationen von den Autorinnen und Autoren nahezu ausnahmslos einen nicht unerheblichen Druckkostenschuss im mittleren vierstelligen Bereich verlangen. ${ }^{10}$ Das Argument der finanziell lukrativen Verlagspublikationen entfällt im Bereich der Doktorarbeiten also. Zudem sind Dissertationen keine klassischen Verlagserzeugnisse. Noch bis in die 1970er Jahre hinein war die Verlagsdissertation eher die Ausnahme. ${ }^{11}$ Die meisten Arbeiten wurden auf Kosten der Autorin bzw. des Autors in einer Druckerei oder später in einem Copy-Shop vervielfältigt und über den Hochschulschriftentausch durch die Universitätsbibliotheken verbreitet. Von daher wäre eine frei zugängliche digitale Publikation über einen Hochschulschriftenserver im Grunde nichts Neues, sondern nur die Rückkehr zu alten Verhältnissen.

Trotzdem erscheinen heute nahezu alle juristischen Dissertationen in Verlagen. Wenn es keine finanziellen Vorteile bietet, in einem Verlag zu publizieren, müssen andere Gründe den Ausschlag dafür geben, auf die grenzenlose Sichtbarkeit einer Online-Publikation zugunsten einer doch eher kleinen Verlagsauflage zu verzichten. Es könnte ja sein, dass Verlage etwa über qualitätsgesicherte Schriftenreihen und damit verbundene renommierte Herausgeberschaften für die fachimmanente Sichtbarkeit einer Arbeit besonders vorteilhaft sind, ${ }^{12}$ so wie eine Publikation, die bei Beck-Online verfügbar ist, die relevante Leserschaft vermutlich zuverlässiger erreicht, als ein noch so brillantes Papier, das zwar frei zugänglich, aber von der Fachöffentlichkeit unbemerkt bloß auf einem Fachrepositorium im Internet veröf-

9 Eine Expertensuche mit der Anfrage „hsg=340 and diss" and jhr=2018 and geo=XA-DE“ im Kata$\log$ der Deutschen Nationalbibliothek ergibt für das Jahr 2018 bei den deutschen juristischen Dissertationen insgesamt 1214 Bücher, aber nur 79 frei zugängliche Online-Ressourcen. In einem durchaus buchaffinen Fach wie Geschichte („hsg=900“) sind es bei den Dissertationen demgegenüber 60 Bücher und 44 frei zugängliche Quellen. Die Aussage von Beyerbach, Die juristische Doktorarbeit - ein Ratgeber für das gesamte Promotionsverfahren, 2. Aufl. München 2017, Rn. 597, dass nur „wenige Fakultäten“ eine elektronische Publikation erlauben, ist nicht nachvollziehbar. Eine Stichprobe der ersten zehn Mitglieder des DJFT in alphabetischer Reihenfolge hat ergeben, dass die jeweiligen Promotionsordnungen ausnahmelos eine elektronische Publikation gestatten. Die einzige dem Verfasser bekannte Ausnahme ist die Juristische Fakultät in Kiel. Der erst jüngst erschienene Ratgeber von Brandt, Dr. jur. - Wege zu einer erfolgreichen Promotion, Baden-Baden 2018, erwähnt in dem Abschnitt über die Veröffentlichung in Rn. 289-305 die Möglichkeit einer elektronischen Dissertation noch nicht einmal.

10 Nach Beyerbach, Die juristische Doktorarbeit - ein Ratgeber für das gesamte Promotionsverfahren, 2. Aufl. München 2017, Rn. 603 sind zwischen 2.000 und $5.000 €$ zu zahlen.

11 Vgl. Leisten, „Grundsätze im Wandel“ - zur Ablösung der KMK-Grundsätze vom 21. November 1974 für die Veröffentlichung der Dissertation durch die revidierende Neufassung vom 28./29. April 1977, in: Jung/Kaegbein (Hrsg.), Dissertationen in Wissenschaft und Bibliotheken, München [u.a.] 1979, S. 79 geht von einer Verlagsquote von Dissertationen über alle Fächer von 16\% aus. Bei den juristischen Dissertationen liegt sie ausweislich der Deutschen Nationalbibliographie derzeit bei über $90 \%$.

12 Auf die Bedeutung „renommierter Schriftenreihen“ stellt auch Beyerbach, Die juristische Doktorarbeit - ein Ratgeber für das gesamte Promotionsverfahren, 2. Aufl. München 2017, Rn. 600 ab. 
fentlicht wird. ${ }^{13}$ Allein schon die vielen Volltextverlinkungen in wichtigen, ebenfalls online verfügbaren Kommentaren sorgen hier für einen enormen Sichtbarkeitsvorsprung der Verlagsveröffentlichung gegenüber einer frei zugänglichen, aber unvernetzten Internetpublikation.

\section{B. Geringe Rezeption von Dissertationen}

Ob eine solche Sichtbarkeit auch bei den juristischen Dissertationen in gleicher Weise gegeben ist, kann jedoch bezweifelt werden. Auffällig ist, dass diese Literaturgattung in Aufsätzen und in der Rechtsprechung nur selten zitiert wird. ${ }^{14}$ Auch in der Kommentarliteratur beschränkt sich ihre Berücksichtigung meist auf einen bibliographischen Nachweis in einer üppig wuchernden und damit meist wenig beachteten Schrifttumsübersicht. Das legt die Vermutung nahe, dass diese Arbeiten auch von Kommentatorinnen und Kommentatoren gar nicht gelesen, sondern nur nach Verlagsprospekt oder auf Grundlage von bibliographischen Verzeichnissen wie der Karlsruher Juristischen Bibliographie (KJB) oder der nützlichen Übersicht neuer Literatur in der Juristenzeitung (JZ) nachgewiesen werden. Immerhin erlangen Verfasserinnen und Verfasser juristischer Aufsätze oder Urteile durch die Angabe im Kommentar Kenntnis von der Existenz einer bestimmten Arbeit. Das bedeutet aber noch nicht, dass dieses Werk auch inhaltlich rezipiert wird. Dafür nämlich muss die Dissertation tatsächlich am Arbeitsplatz juristischer Autorinnen und Autoren verfügbar sein. Angesichts der nicht geringen Preise im Buchhandel, die leicht 50 bis $70 €$ erreichen, aber auch deutlich darüber liegen können, ist für die Erreichbarkeit juristischer Dissertationen ihr Vorhandensein im Bestand der örtlichen Bibliothek von zentraler Bedeutung.

Hier darf man erwarten, dass juristische Dissertationen an Standorten mit Juristischen Fakultäten regelmäßig und zuverlässig zu finden sind. Nimmt man die Mitglieder des Deutschen Juristen-Fakultätentages (DJFT) als Grundlage, so gibt es in Deutschland derzeit 45 Juristische Fakultäten bzw. Fachbereiche oder Hochschulen mit der Berechtigung, einen Doktor der Rechte zu verleihen. ${ }^{15}$ Diese Zahl ist eine gute Orientierungsgröße, um die Verbreitung juristischer Dissertationen und damit auch die Chance, dass sie gelesen und im Fachdiskurs berücksichtigt werden, abschätzen zu können. Zwar besteht immer die Möglichkeit, auch sehr gering verbreitete Titel über die Fernleihe zu beschaffen, doch wird dieser Weg nur bei einem wirklichen und konkreten Interesse beschritten. In der bibliothekarischen Praxis ist

13 Die mangelnde Sichtbarkeit im „großen Rauschen“ des Internet ist für von Münch/Mankowski, Promotion, 4. Aufl., Tübingen 2013, 174 f. ein Argument gegen eine bloß elektronische Publikation von Dissertationen.

14 Die geringe Rezeption von Monographien, bei denen zahlenmäßig die Dissertationen den Löwenanteil ausmachen, beklagt auch Wissenschaftsrat, Perspektiven der Rechtswissenschaft in Deutschland - Situation, Analysen, Empfehlungen, Köln 2012, S. 66 f.

15 Vgl. die Selbstdarstellung des DJFT unter https://www.djft.de/der-djft/ (Abruf am 23. April 2019). 
zu beobachten, dass vor Ort nicht vorhandene Titel eher übergangen als über den auswärtigen Leihverkehr angefordert werden. Hier spielt nicht nur der zunehmende Zeitdruck beim Abfassen eigener wissenschaftlicher Texte eine Rolle, sondern auch die durch eine Volltextdatenbank wie Beck-Online kultivierte Erwartungshaltung, auf einen gewünschten Inhalt sogleich zugreifen zu können. Wie steht es aber nun um die Vor-Ort-Verfügbarkeit von juristischen Dissertationen? Zwei kleine Erhebungen auf Grundlage der über das Internet frei zugänglichen Online-Bibliothekskataloge sollen hier eine beispielhafte Antwort geben. ${ }^{16}$

\section{Die Verbreitung Hagener juristischer Dissertationen in Bibliotheken}

Im Rahmen einer internen Evaluation der Universitätsbibliothek Hagen wurde die Verbreitung der in den Jahren 1997 bis 2010 an der Rechtswissenschaftlichen Fakultät abgeschlossenen Dissertationen untersucht. Es handelt sich um insgesamt 120 Arbeiten. Im Schnitt sind diese Arbeiten an 21 Bibliotheksstandorten zu finden. Nur drei Arbeiten stehen in mehr als 50 Bibliotheken, 22 Arbeiten jedoch sind an weniger als zehn Standorten verfügbar. Hier ist zu berücksichtigen, dass die Bibliotheken mit Hagener Dissertationen nicht nur juristische Fakultätsbibliotheken oder Universitätsbibliotheken sind, sondern auch Staats- oder Landesbibliotheken sowie große Gerichtsbibliotheken. Mit Blick auf den juristischen Fachdiskurs sind aber gerade Standorte mit einer Fakultät von Bedeutung, denn hier werden nicht nur neue und damit in nicht wenigen Fällen auch thematische weiterführende Dissertationen geschrieben, hier entstehen auch wichtige Aufsätze, Handbücher und Kommentare. Ausgehend von der Zahl von 45 Fakultäten in Deutschland kann man für die älteren Hagener Dissertationen sagen, dass sie noch nicht einmal an der Hälfte aller Fakultäten verfügbar sind.

Ein besonders markantes Beispiel für die sehr begrenzte Verbreitung einer Dissertation ist die Arbeit von Markus Pein zum Thema „Prozessuale Probleme des Studienplatzverfahrens". ${ }^{17}$ Abgesehen von den beiden Pflichtexemplaren in der Deutschen Nationalbibliothek, ist sie nur an zehn weiteren Standorten verfügbar, darunter befinden sich die Landbibliothek in Kiel mit dem regionalen Pflichtexemplar, die Universitätsbibliothek in Hagen mit dem Pflichtstück aufgrund der Promotionsordnung sowie die für die interessierte Öffentlichkeit nur eingeschränkt zu-

16 Die jeweiligen Dissertationen wurden zunächst durch eine Recherche in der Deutschen Nationalbibliographie über die Kataloge bei der Deutschen Nationalbibliothek bibliogrphisch ermittelt. Die jeweiligen Bibliotheksstandorte wurden dann in den mit einer Suche im Karlsruher Virtuellen Katalog angesteuerten Verbundkatalogen ausgezählt. Bei diesem Verfahren werden Doppelstücke an einem Standort nicht erfasst. Mehrere Institutsbibliotheken an einem Standort wurden ebenfalls nicht separat gezählt, sondern der jeweiligen Universitätsbibliothek zugeschlagen. Für die Zwecke der Erhebung kam es nur auf die Verfügbarkeit in einem bestimmten Bibliothekssystem überhaupt an. Auch nach $\mathbb{S} 1$ Nr. 3 Buchstabe a) und b) der Ordnung des Leihverkehrs in der Bundesrepublik Deutschland (Leihverkehrsordnung) gilt eine solche Verfügbarkeit als lokaler Bestand und schließt eine Fernleihbestellung aus.

17 Markus Pein, Prozessuale Probleme des Studienplatzverfahrens, Tönning [u.a.] 2009, XV, 181 S. 
gänglichen Bibliotheken des Bundesverfassungsgerichts und des Heidelberger MaxPlanck-Instituts für ausländisches öffentliches Recht und Völkerrecht. Übrig bleiben damit ganze sechs Hochschulbibliotheken die diese Arbeit in ihren Bestand aufgenommen haben, nämlich Bonn, Erlangen, Frankfurt am Main, Hannover, Münster und Potsdam. Bedenkt man, dass das Thema dieser Dissertation eigentlich jede Hochschule in Deutschland interessieren sollte und dass der Ladenpreis des Buches mit 29,90 € für eine Dissertation erstaunlich niedrig war, wundert dieses Ergebnis umso mehr. Angemerkt sei, dass die Arbeit von Pein als Prädikatsdissertation mit einem Promotionspreis ausgezeichnet wurde. ${ }^{18}$ Das macht die sehr geringe Verbreitung noch bedauerlicher.

\section{Die Verbreitung Frankfurter juristischer Dissertationen in Bibliotheken}

In Vorbereitung auf die Tagung „Open Access in der Rechtswissenschaft“ am 18. und 19. Oktober 2018 an der Goethe-Universität in Frankfurt am Main wurden die Frankfurter rechtswissenschaftlichen Dissertationen des Jahres 2015 untersucht. ${ }^{19}$ Es kann davon ausgegangen werden, dass für diese Arbeiten drei Jahre nach ihrer Veröffentlichung nur noch sporadisch neue Erwerbungsentscheidungen getroffen werden, so dass die jetzt vorliegende Verbreitung als im Wesentlichen endgültig betrachtet werden kann. In der Reihe H der von der Deutschen Nationalbibliothek erstellten Deutschen Nationalbibliographie, die die Hochschulschriften verzeichnet, wurden 2015 insgesamt 32 rechtswissenschaftliche Dissertationen der Frankfurter Goethe-Universität nachgewiesen. Von diesen Arbeiten sind 31 in einem Verlag erschienen, lediglich eine Dissertation wurde über den Tausch der Universitätsbibliothek als reine Hochschulschrift verbreitet. Keine dieser Arbeiten ist frei zugänglich im Sinne von Open Access, doch werden einige Titel von Verlagen parallel zur Buchausgabe auch als kostenpflichtiges eBook angeboten. Diese eBooks stehen meist im Rahmen größerer eBook-Pakete in einem authentifizierten Zugriff den Angehörigen einzelner Hochschulen zur Verfügung. Als Ergebnis der Sichtung der einschlägigen Bibliothekskataloge ist festzuhalten, dass die 32 Frankfurter Dissertationen im Schnitt in 20 Bibliotheken als gedrucktes Buch zu finden sind. Dieser Befund deckt sich mit der in Hagen gemachten Beobachtung. Nimmt man jetzt noch die eBooks in größeren Paketen hinzu, so erhöht sich diese Zahl auf im Schnitt 25 Standorte. Damit würde rein zahlenmäßig knapp die Hälfte der Juristischen Fakultäten mit den Frankfurter Dissertationen des Verzeichnungsjahres 2015 versorgt sein.

Im Gegensatz zu den Hagener Arbeiten wurden die Frankfurter Dissertationen noch einmal sehr genau nach Fakultätsstandorten untersucht und dabei auch Dop-

18 Vgl. FernUni Perspektive - Zeitung für Angehörige, Freundinnen und Freunde der Fernuniversität, Ausgabe Nr. 30 (Winter 2009), S. 2.

19 Vgl. den Tagungsbericht von Wildgans, Zuckerbrot oder Peitsche? - Ein Plädoyer für Open Access im juristischen Publikationswesen, in: ZUM 2019, S. 21-28. 
pelungen eines gedruckten und parallelen elektronischen Zugangs ausgeschlossen. Hier zeigt es sich, dass die Frankfurter Arbeiten durchschnittlich an nur 16 Standorten mit einer Juristischen Fakultät zu finden waren. Zehn der 32 Arbeiten waren sogar an weniger als zehn Fakultätsorten vertreten, davon zwei Dissertationen sogar nur an drei Universitäten, Frankfurt selbst schon miteingerechnet. Das Ergebnis ist recht ernüchternd. Obwohl die Arbeiten professionell über den Verlagsbuchhandel vertrieben werden, sind sie an 2/3 der Juristischen Fakultäten nicht vor Ort verfügbar.

Das Ergebnis der Hagener als auch das Frankfurter Untersuchung ist nicht im strengen Sinn repräsentativ. Es zeigt aber einen Trend, der sich auch an anderen Orten und in der bibliothekarischen Praxis gut beobachten lässt, nämlich dass juristische Dissertationen in den Bibliotheken eher mäßig, keinesfalls aber flächendeckend verbreitet sind und gesammelt werden. ${ }^{20}$ Angesichts des gar nicht so seltenen Falles von Titeln, die an weniger als zehn Standorten vorgehalten werden, stellt sich die Frage, ob damit der für Dissertationen geltenden Publikationspflicht überhaupt noch effektiv entsprochen wird.

\section{E. Die Pflicht zur angemessenen Veröffentlichung}

In allen Promotionsordnungen finden sich Vorschriften zur Veröffentlichung von Dissertationen. Historisch soll mit dieser Veröffentlichungspflicht zweierlei bezweckt werden, nämlich erstens die akademische Integrität des Promotionswesens zu sichern und zweitens den wissenschaftlichen Erkenntnisfortschritt im Fach zu fördern. ${ }^{21}$ Zur Sicherung der akademischen Integrität ist die Zugänglichkeit für die allgemeine Öffentlichkeit erforderlich, um im Bedarfsfall die wissenschaftliche Güte, die Originalität und die methodisch saubere Arbeitsweise bei einer bestimmten Arbeit überprüfen zu können. Zu diesem Zweck reichen bereits wenige, in öffentlichen Bibliotheken leicht zugängliche Exemplare aus. ${ }^{22}$ Soll darüber hinaus jedoch

20 Dieser Befund ist nicht auf das Fach Rechtswissenschaft beschränkt und keinesfalls neu. Babendreier, Dissertationentausch - Vervielfältigung, Verbreitung und Archivierung von Hochschulschriften im elektronischen Zeitalter, in: ABI-Technik 23 (2003), S. 20 f. spricht hier von einem „rigiden Selektionsprozess“. Allerdings bezieht er sich auf die über den Tausch verbreiteten Dissertationen. Dieser Erwerbungsweg spielt jedenfalls im juristischen Fachreferat in der Praxis keine Rolle. Zustimmend kann mit man Babendreier darin aber ein Desinteresse der Bibliotheken an dieser Litertaturform beobachten, das freilich nur ein Reflex einer mangelnden Nachfrage ist. Sich damit abzufinden, bedeutet freilich, Dissertationen als Forschungsleistung abzuwerten. Die mangelnde Verbreitung von Dissertationen kritisiert auch schon Lohse, Zur qualitativen Bedeutung des Zugangs Tausch/Geschenk, in: Mitteilungsblatt (MB) 37 (1987), S. 165 f.

21 Vgl. Babendreier, Dissertationentausch - Vervielfältigung, Verbreitung und Archivierung von Hochschulschriften im elektronischen Zeitalter, in: ABI-Technik 23 (2003), S. 16.

22 Vgl. dazu am Beispiel der österreichischen Rechtslage Staudegger, Open-Access-Veröffentlichungspflicht für Dissertationen? - Eine rechtswissenschaftliche Untersuchung aus Anlass der Ergänzung von $\mathbb{S} 86$ Abs 1 UG durch BGBl.I 2017/129, in: Austrian Law Journal 2018, S.9f. (DOI:10.25364/01.5:2018.1.1). 
auch der wissenschaftliche Erkenntnisfortschritt gefördert werden, ${ }^{23}$ so muss eine angemessene Sichtbarkeit im akademischen Diskurs gegeben sein, die nicht nur eine bloße Zugänglichkeit, sondern auch eine gewisse Verbreitung innerhalb der Fachöffentlichkeit voraussetzt. ${ }^{24}$

Im diesem Sinne bestimmen auch die Grundsätze für die Veröffentlichung von Dissertationen der Kultusministerkonferenz, dass eine solche Arbeit „in angemessener Weise der wissenschaftlichen Öffentlichkeit durch Vervielfältigung und Verbreitung zugänglich zu machen“ ist. Was in diesem Sinne als angemessen zu verstehen ist, wird dahingehend konkretisiert, dass in den Geistes- und Gesellschaftswissenschaften, wozu auch die Rechtswissenschaft zu zählen ist, bis zu 80 Exemplare für den Schriftentausch abzuliefern sind. Bei einer Zahl von bis zu 80 Exemplaren kann tatsächlich jeder Fakultätsstandort mit einem Exemplar versorgt werden. Alternativ ist nach den Grundsätzen der Kultusministerkonferenz auch eine Verlagspublikation zulässig, wenn die Mindestauflage 150 Exemplare beträgt. Durch das Erfordernis einer Mindestauflage soll offenbar eine der Vertauschung von bis zu 80 Exemplaren vergleichbare Verbreitungswirkung erreicht werden. Als weitere Optionen ist noch die Mikroform mit immerhin 50 Kopien oder die Online-Dissertation mit ihrer im Prinzip unbeschränkten Verbreitung vorgesehen.

Angesichts der recht konkreten Zahlen der Kultusministerkonferenz können 20 bis 25 Bibliothekstandorte oder bloß 1/3 der Juristischen Fakultäten wohl kaum als angemessene Verbreitung gelten. Man fragt sich auch, wo bei einer Mindestauflage von 150 Exemplaren die übrigen 120 bis 130 meist hochpreisigen Stücke abgesetzt und verbreitet werden sollen, wenn Bibliotheken als institutionelle Käufer weitgehend ausfallen? Auch das Leitbild von bis 80 Tauschexemplaren wird eindrucksvoll verfehlt. Ohne sogleich über Open Access und damit eine frei zugängliche Publikation im Internet sprechen zu wollen, darf angesichts der nicht geringen Druckkostenzuschüsse für Verlagspublikationen schon gefragt werden, ob nicht für den gleichen Betrag vielleicht 50 Tauschexemplare in einem Copy-Shop hergestellt und anschließend über die Bibliotheken effektiver verbreitet werden könnten. Die Verfügbarkeit und damit auch die Sichtbarkeit von Dissertationen würde allein schon durch diese Maßnahme für die Autorinnen und Autoren kostenneutral verdoppelt, von den ersparten Erwerbungsmitteln bei den Bibliotheken gar nicht zu reden. Tatsächlich hat die Frage des Verhältnisses von Tauschdissertation und Ver-

23 Bei der Ablieferung von Tauschexemplaren an eine Bibliothek ist dies der Hauptzweck der Verpflichtung, vgl. Kobusch, Pflichtexemplare einer Dissertation im Lichte des Urhebergesetzes, in: Wissenschaftsrecht 34 (2001), S. 264.

24 Babendreier, Dissertationentausch - Vervielfältigung, Verbreitung und Archivierung von Hochschulschriften im elektronischen Zeitalter, in: ABI-Technik 23 (2003), S. 16 f. 
lagspublikation viele Jahrzehnte die Diskussion um die angemessene Publikation von Dissertationen bestimmt. ${ }^{25}$

\section{F. Die Dissertation als bibliothekarischer Ballast}

Aus bibliothekarischer Sicht wurde die verlegerische Publikation von Dissertationen vor allem deshalb sehr kritisch gesehen, weil sie den Erwerbungsetat unnötig belastete. Traditionell nämlich wurden diese Arbeiten außerhalb des Buchhandels vervielfältigt und dann in hoher, nicht selten dreistelliger Stückzahl an die Universität zur weiteren Verbreitung im Wege des Tauschs abgeliefert. Eine wichtige Grundlage für diesen Tausch war für die preußischen Universitäten der „Ministerial-Erlaß vom 12. September 1913 betreffend die Versendung und Verzeichnung der an den preußischen Universitäten und Technischen Hochschulen erscheinenden Schriften ". ${ }^{26}$ Er sah vor, dass die akademischen Schriften von Universitäten und Fakultäten in ausreichender Zahl an die jeweilige Universitätsbibliothek abzuliefern waren. Die Bibliotheken tauschten dann diese Schriften untereinander regelmäßig aus. So wurde sichergestellt, dass jede Dissertation an jedem Standort tatsächlich verfügbar war. ${ }^{27}$ Mit dem zunehmenden Aufkommen von Verlagsdissertationen wurde die Tauschpraxis in Frage gestellt. Immerhin konnten sich Bibliotheken und Buchhandel 1937 darauf verständigen, dass auch von einer Verlagsdissertation bis zu 50 Exemplare für den Schriftentausch zur Verfügung stehen sollten. ${ }^{28}$

Diese Regelung war für die Bibliotheken recht günstig und für die Verlage nicht unbedingt schädlich, da die Kosten für die Tauschexemplare von den Autorinnen und Autoren zu bestreiten waren, was bei Licht besehen einen sicheren Absatz für den Verlag bedeutete. Von den Autorinnen und Autoren wurden die damit verbundenen hohen Kosten als eine erhebliche Belastung empfunden. Es fehlte nicht an Stimmen in der Literatur, die hier eine Verletzung des Grundrechts aus Art 14 GG sahen. ${ }^{29}$ In der Folgezeit wurden die abzuliefernden Verlagsdissertationen in den Promotionsordnungen immer weiter reduziert. ${ }^{30}$ Die schon erwähnten Grundsätze

25 Vgl. dazu auch mit historischen Hinweisen Stois, Die Rechtsgrundlagen des akademischen Schriftentausches, in: Zentralblatt für Bibliothekswesen (52) 1935, S. 53-85.

26 Abgedruckt in: Jahrbuch der Deutschen Bibliotheken 12 (1914), S. 179-181.

27 Babendreier, Dissertationentausch - Vervielfältigung, Verbreitung und Archivierung von Hochschulschriften im elektronischen Zeitalter, in: ABI-Technik 23 (2003), S. 19; Leyh, Spannungen und Lösungen im akademischen Schriftentausch, in: ZfB 58 (1941), S. $261 \mathrm{f}$.

28 Die Vereinbarung ist abgedruckt in: Zentralblatt für Bibliothekswesen 55 (1938), S. 71.

29 So im Ergebnis Walter, Dissertations-Druckzwang und Grundgesetz, in: DVBl. 1972, S. 309-312.

30 Eindrücklich ist hier die Promotionsordnung (Satzung) der Rechtswissenschaftlichen Fakultät der Christian-Albrechts-Universität zu Kiel vom 27. März 2017 (Fundstelle: (NBl. HS MSGWG Schl.-H., S. 28). Sie kennt nicht nur als seltene Ausnahme unter den deutschen Fakultäten keine elektronische Dissertation, sondern fordert in ihrem $\$ 25$ auch noch 80 Pflichtexemplare. Wird die Arbeit in einem Verlag publiziert, reduziert sich diese Zahl auf acht. Vorschriften wie diese waren in der Vergangenheit ein Grund, für die Ausweitung der Quote der Verlagsdissertationen, dazu auch Leisten, „Grundsätze im Wandel“ - zur Ablösung der KMK-Grundsätze vom 21. November 1974 für die Veröffentlichung der Dissertation durch die revidierende Neufassung vom 28./29. April 
der Kultusministerkonferenz gehen auf diesen Punkt gar nicht ein und stellen ihn damit vollständig in das Ermessen der jeweiligen Universität bzw. Fakultät vor Ort.

Vergleicht man die historische Tauschpraxis, die ja auf eine Vollversorgung an jedem Standort ausgerichtet war, mit der aktuellen Verbreitungssituation von juristischen Dissertationen, so kann man kaum noch von einer angemessenen Verbreitung in der wissenschaftlichen Öffentlichkeit sprechen. Arbeiten, die an weniger als zehn Standorten zugänglich sind, verfehlen dieses Erfordernis sicher. ${ }^{31}$

Es wäre aber zu kurz gegriffen, das Problem der mangelnden Sichtbarkeit und Verbreitung allein darauf zurückzuführen, dass Bibliotheken wegen unzureichender Finanzierung möglicherweise nicht in der Lage sind, die ihnen nun nicht mehr im Wege des Tauschs unberechnet zufließenden Arbeiten käuflich zu erwerben. Sieht man von dem im Vergleich zum Tausch ungleich größeren Personalaufwand bei der Erwerbung dieser Arbeiten über den Handel einmal ab, so darf eine wichtige, ja entscheidende Größe nicht aus dem Blick geraten, nämlich die Stellfläche im Regal und Magazin. ${ }^{32}$ Bei konservativer Schätzung können auf einem Meter Regalfläche rund 55 Arbeiten Platz finden. Jährlich verzeichnet die Deutsche Nationalbibliothek aber wenigstens 1.300 juristische Dissertationen. Daraus ergibt sich ein Platzbedarf von gut 23 Metern pro Jahr allein für die Rechtswissenschaft! Ein solcher Zuwachs lässt sich aus Platzgründen nicht mehr seriös verwalten!

Dissertationen sind daher wenigstens seit den 70er Jahren eine für die Bibliotheksplanung problematische Literaturgattung. Eine prominente Empfehlung des Wissenschaftsrats propagierte damals für Universitätsbibliotheken das Leitbild eines Nullwachstums bzw. einer Bestandsobergrenze. ${ }^{33}$ Das bedeutet, dass Neuzugang und Aussonderung sich in etwa die Waage halten sollten. Auch wenn sich aus überlieferungsgeschichtlicher Sicht viel gegen diese Vorgaben einwenden lässt, die Tatsache, dass neue Magazinflächen mit erheblichem finanziellen und administrativen Aufwand verbunden sind und damit vielfach nicht zur Verfügung stehen,

1977, in: Jung/Kaegbein (Hrsg.), Dissertationen in Wissenschaft und Bibliotheken, München [u.a.] 1979, S. 79.

31 Vgl. Schnieders, Rechtsfragen im Bereich von Promotion und Dissertation, in: Jung/Kaegbein (Hrsg.), Dissertationen in Wissenschaft und Bibliotheken, München [u.a.] 1979, S. 101, der ebenfalls eine Verbreitung von zehn Exemplaren für zu wenig hält und eine angemessene Verbreitung bei 150 Exemplaren als gegeben ansieht. Diese Zahl entspricht auch der aktuell von der KMK bei Verlagsdissertationen geforderten Mindestauflage.

32 Vgl. Babendreier, Dissertationentausch - Vervielfältigung, Verbreitung und Archivierung von Hochschulschriften im elektronischen Zeitalter, in: ABI-Technik 23 (2003), S. 18.

33 Wissenschaftsrat, Empfehlungen zum Magazinbedarf wissenschaftlicher Bibliotheken, Köln 1986, S. 36 f. Siehe auch Bonte, Die Empfehlungen der HIS GmbH zur Organisation und Ressourcenplanung für wissenschaftliche Bibliotheken, in: ABI-Technik 26 (2006), S. 32; Tannhof, „Nullwachstum “ für die Hochschulbibliotheken? : Zum Stand der Diskussion um die Empfehlungen des Wissenschaftsrates zum Magazinbedarf wissenschaftlicher Bibliotheken, in: Arbido-R 2 (1987), S. 65-70. 
zwingt Bibliotheken zu Maßnahmen der Bestandsreduktion. Für die Dissertationen bedeutete dies zunächst eine Verringerung des Tauschs, um den Neuzugang einzudämmen. Zugleich wurden ältere Arbeiten mit geringer Benutzung in weitem Umfang ausgesondert. Ausgenommen waren davon lediglich die Arbeiten der eigenen Hochschule. ${ }^{34}$ Was 1914 mit einer flächendeckenden Versorgung durch den Schriftentausch gestartet ist, endete in der Zeit eines expansiven Hochschulausbaus nicht selten mit einer forcierten Makulierung der vor Ort praktisch ungenutzten Titel. Verschärft wurde das Mengenproblem durch die Neugründung von Fakultäten und die steigenden Studierendenzahlen, wodurch auch die Zahl der Dissertationen gegenüber der Vorkriegszeit enorm zugenommen hat. ${ }^{35}$ Wenn man gleichwohl noch am Ideal einer umfassenden Verfügbarkeit dieser Arbeiten festhalten wollte, so galt es, neue Wege der Verbreitung und Aufbewahrung zu beschreiten. Hier wurden vor allem in die Mikroform hohe Erwartungen gesteckt. ${ }^{36}$ War auch die Platzersparnis gegenüber dem gedruckten Buch enorm, so hat sich die Mikroform bei den Nutzern nie wirklich durchgesetzt und ist bis heute nur ein Nischenprodukt geblieben.

\section{G. Die elektronische Dissertation}

Das kann von der elektronischen Dissertation jedenfalls auf der Nutzerseite nicht gesagt werden. Gerade wegen der schnellen Verfügbarkeit unmittelbar am Arbeitsplatz, der Möglichkeit einer Volltextsuche sowie der digitalen Weiterverarbeitung von Zitaten werden digitale Dissertationen, wenn sie denn verfügbar sind, gerne genutzt. Wo beispielsweise Bibliotheken umfangreiche eBook-Pakete mit Dissertationen erwerben, gibt es praktisch keine Anschaffungsvorschläge mehr für gedruckte Exemplare. Auch wenn das traditionelle Buch für die intensive Lektüre umfangreicher Text immer noch als unentbehrlich bezeichnet wird, aus der Bibliothekspraxis lässt sich dieses Bild so nicht bestätigen. Offenbar werden Dissertationen ohnehin nur kursorisch und punktuell rezipiert, was im digitalen Format ohne Weiteres leicht und dank der Volltextsuche auch effektiv möglich ist. Wenn eine Arbeit wirklich intensiv durchgearbeitet wird, wird das Buch privat erworben, weil dann auch Anmerkungen notiert werden können. Diese Ehre dürfte aber nur wenigen herausragenden oder für die eigene Forschung besonders einschlägigen Arbeiten zuteilwerden.

34 Vgl. beispielhaft den Bericht über eine Evaluation von 210.000 Dissertationen in der Universitätsbibliothek Freiburg „Rückführung der Dissertationen aus dem Landesspeicher in Karlsruhe“, in: Expressum - Informationen aus dem Freiburger Bibliothekssystem 2000, Heft 2, S. 22 f.

35 Für den Zeitraum von 1968 bis 1994 kommt Schroeder, Die Entwicklung der juristischen Promotionen in Deutschland, in: JuS 1998, S. 281 fast auf eine Verdoppelung der Anzahl der Dissertationen im Fach Rechtswissenschaft.

36 Wissenschaftsrat, Empfehlungen zum Magazinbedarf wissenschaftlicher Bibliotheken, Köln 1986, S. 25 f.; Babendreier, Dissertationentausch - Vervielfältigung, Verbreitung und Archivierung von Hochschulschriften im elektronischen Zeitalter, in: ABI-Technik 23 (2003), S. 18. 
Trotz dieser Vorteile auf der Nutzerseite hat sich bei den Doktorandinnen und Doktoranden die elektronische Dissertation als Form jedenfalls in der Rechtswissenschaft nicht durchgesetzt. Die vielen, vor allem in eBook-Paketen verfügbaren Titel werden allein auf Betreiben der Verlage zusätzlich zum gedruckten Buch angeboten, weil insbesondere die Bibliotheken u.a. auch aus Gründen der Regalkapazität und der Nutzerfreundlichkeit diese Ausgabeform gerne erwerben. Die Autorinnen und Autoren hingegen legen immer noch großen Wert darauf, nach dem erfolgreichen Abschluss der Promotion ein „richtiges“ Buch in der Hand zu halten. Das gleiche gilt auch für die intensive Lektüre bei den wenigen echten Leserinnen und Leser einer juristischen Dissertation.

Halten wir an dieser Stelle kurz inne. Es wurde festgestellt, dass derzeit juristische Dissertationen nur unzureichend in Fachbibliotheken verfügbar sind. In den allermeisten Fällen kann man nicht mehr von einer angemessenen Verbreitung in der wissenschaftlichen Öffentlichkeit sprechen kann. Vordergründig scheint dies ein Problem der Verlagsdissertation zu sein, die im Gegensatz zu den außerhalb des Buchhandels erschienenen Arbeiten nicht mehr automatisch an alle Fakultäten geschickt wird. ${ }^{37}$ Tatsächlich aber sind Bibliotheken allein wegen der schieren Menge rechtswissenschaftlicher Doktorarbeiten organisatorisch gar nicht in der Lage, alle oder wenigstens die meisten dieser Arbeiten kontinuierlich in ihren Bestand zu übernehmen und dauerhaft vorzuhalten. Eine Ausweitung der Sammlung gedruckter Dissertationen ist daher kein Weg, um die mangelnde Verbreitung und damit auch Sichtbarkeit dieser Arbeiten zu verbessern. Hier verspricht allein die digitale Form Abhilfe. Jedenfalls auf Seiten der Nutzerinnen und Nutzer wird diese Art des Angebots bereits recht gut angenommen, zumal neben einer schnellen Bereitstellung des Inhalts auch digitale Mehrwerte geboten werden.

Allerdings sind diese digitalen Dissertationen nicht frei zugänglich im Sinne von Open Access. Sie stehen nur angemeldeten Bibliotheksnutzerinnen und Bibliotheksnutzern in einem authentifizierten Zugriff zur Verfügung und müssen vorher von den Bibliotheken erworben werden. Zwar können Bibliotheken diese Arbeiten auch einzeln lizenzieren, doch wird aus Gründen der Arbeitsökonomie meist dem Erwerb in Paketen der Vorzug gegeben, wenn der Bibliotheksetat diese recht kostspielige Form der Anschaffung erlaubt. Gerade kleine Fakultäten werden sich dies nicht in vollem Umfang leisten können. Zudem ermöglicht die digitale Form der

37 Die Aussage von von Münch/Mankowski, Promotion, 4. Aufl., Tübingen 2013, 174 f. dass eine Online-Dissertation in vielen Fächern am wissenschaftlichen Diskurs nicht teilnimmt und ein Buch, das nicht in einem Verlag erscheint, nicht existiert, ist nur bedingt richtig. Empirisch gesehen sind juristische Verlagsdissertationen in den meisten Bibliotheken inexistent. Richtig ist freilich, dass allein sie eine reelle Chance hat, im Rahmen einer Rezension gewürdigt zu werden. Dass elektronische Dissertationen nicht am Diskurs teilnehmen, ist eine These, die sicher diskussionswürdig ist. Vorrangiger Untersuchungsgegenstand wäre dann freilich die Informationskompetenz juristischer Autorinnen und Autoren. 
Nutzung eine sehr transparente Nutzungsanalyse. Während in einer traditionellen Bibliothek jenseits von Ausleihstatistiken kaum nachgeprüft werden kann, ob Arbeiten am Regal eingesehen oder anderweitig innerhalb der Bibliothek genutzt werden, zeigen digitale Plattformen ein sehr präzises Abbild der tatsächlichen Nutzungen. Und hier ist es häufig zu beobachten, dass von mitunter mehreren hundert erworbenen Dissertationen viele Arbeiten nie oder nur sehr wenig zur Kenntnis genommen werden. Es stellt sich dann durchaus die Frage, ob die knappen Erwerbungsmittel beim Kauf von großen eBook-Paketen mit juristischen Dissertationen tatsächlich wirtschaftlich eingesetzt werden. Manche Verlage habe auf diese Entwicklung bereits mit Kaufmodellen reagiert, bei denen praktisch alle lieferbaren Titel zur Recherche zur Verfügung gestellt werden, ein Kauf aber erst dann erfolgt, wenn diese Titel auch tatsächlich genutzt werden. ${ }^{38}$ Auf diesem Wege stehen sehr viele Arbeiten sogleich zur Verfügung, ohne dass sie in jedem Fall auch wirklich erworben werden müssen. Liegt hier schon eine Lösung für das eingangs beschriebene Verbreitungsproblem juristischer Dissertationen? Könnte die Zukunft so aussehen, dass im Prinzip alle Arbeiten über entsprechende Plattformen verfügbar sind, aber erst im Nutzungsfall erworben werden? Ein solches Modell könnte tatsächlich die aktuelle Versorgungslage im Umfeld Juristischer Fakultäten entscheidend verbessern. Die Frage ist aber, ob dieses Modell wirklich eine Zukunft hat.

\section{H. Adressatenkreis von juristischen Dissertationen}

Im Gegensatz etwa zu sprachwissenschaftlichen Arbeiten, die ihr Publikum im Wesentlichen in wissenschaftlichen Einrichtungen haben, können juristische Dissertationen ganz unterschiedliche Zielgruppen außerhalb der akademischen Rechtswissenschaft im engeren Sinn interessieren. Man denke nur an die sehr zahlreiche Anwaltschaft, aber auch an die Justiz, die öffentliche Verwaltung und die Politik bzw. Gesetzgebung. Auch über den engen Kreis der Rechtswissenschaft hinaus können juristische Dissertationen für andere Fachdisziplinen von Interesse sein. Verfassungsrechtliche Arbeiten etwa haben auch eine Leserschaft in der Politikwissenschaft, steuerrechtliche Arbeiten können die Wirtschaftswissenschaften interessieren, Arbeiten aus den Grundlagenfächern die Sozialwissenschaften oder die Geschichte. Es lassen sich leicht viele weitere Beispiele für interdisziplinäre Fragestellungen finden. Es ist fraglich, ob diese vielen unterschiedlichen Zielgruppen über zu lizenzierende digitale Verlagsangebote tatsächlich erreicht werden. Für andere Wissenschaftsfächer an Universitäten mit einer Juristischen Fakultät mag das der Fall sein, für die Anwaltschaft, die Justiz und die Politik eher nicht. Dabei behan-

38 Das ist bei dem so genannten Patron Driven Acquisiton (PDA) Modell der Fall, das vor allem im Bereich der englischsprachigen Wissenschaftsliteratur angeboten wird, vgl. dazu allgemein Berg/ Hanke, Experimentierfeld oder Paradigmenwechsel? Eine Handreichung der BVB-Kommission für Elektronische Ressourcen, in: Bibliotheksforum Bayern 6 (2012), S. 90-93; Swords (Hrsg.), Patrondriven acquisitions - History and best practices. Berlin 2011. 
deln viele Dissertationen praxisrelevante Themen und bieten Lösungen für die Gerichte und den Gesetzgeber an. Wenn Justiz und Politik juristische Dissertationen aus Gründen beschränkter Zugänglichkeit aber kaum zur Kenntnis nehmen, führt dies auch zu einer inakzeptablen Ineffizienz rechtswissenschaftlicher Forschung. Darauf zu verweisen, dass interessierte Gerichte und Parlamente ja die entsprechenden Bücher erwerben können, überschätzt die bibliothekarische Versorgung speziell der Justiz unterhalb der obersten Gerichtsbarkeit und verkennt zudem die von hohem Zeitdruck geprägte Arbeitsweise in diesen Bereichen. ${ }^{39}$ Was nicht elektronisch sofort erreichbar und vor allem durch allgemeine Suchmaschinen leicht auffindbar ist, wird nicht zur Kenntnis genommen. Solange Dissertationen nur als gedruckte Bücher oder hinter Paywalls in kostenpflichtigen Verlagsdatenbanken zugänglich sein, korrespondiert ihre mangelhafte Verbreitung mit einem verbreiteten Desinteresse. Insoweit könnte man argumentieren, dass doch eigentlich alles gut funktioniere, wenn hochspezielle Forschungsliteratur nur streng nachfrageorientiert angeschafft und damit sehr effizient zugänglich gemacht wird. Dieser Einwand verwechselt freilich die tatsächliche Nachfrage unter unzureichenden medialen Bedingungen mit dem diskursiven Potenzial der in den Dissertationen erhaltenen Forschung. Das gilt vor allem für die juristische Praxis, die anders als über allgemeine Suchmaschinen solche Arbeiten praktisch nicht zur Kenntnis nimmt. Hier liegt ein entscheidener Unterschied zwischen der Forderung eines umfassenden Schriftentausches oder dem Ruf nach mehr Geld und Platz für mehr Verlagsdissertationen und Open Access. Beim Schriftentausch und Bücherkauf werden bloß schlecht erschlossene und im Ergebnis kaum nachgefragte Papierberge angehäuft. Im Gegensatz dazu erschließt Open Access ganz neue Leserkreise

\section{Wer soll das alles lesen?}

Bisher wurde selbstverständlich vorausgesetzt, dass juristische Dissertationen, wenn die wissenschaftliche Öffentlichkeit sie zur Kenntnis nimmt, von Mitgliedern eben dieser Öffentlichkeit auch gelesen werden. Diese Feststellung erscheint trivial, sie ist aber für eine funktionierende Forschungskommunikation in der Rechtswissenschaft nicht ohne Probleme. Hier geht es um die Frage, welche tatsächliche Lesezeit wissenschaftlich arbeitenden Juristinnen und Juristen zur Verfügung steht und welche Lesezeit sie haben müssten, um die für ihr Forschungsgebiet einschlägigen Arbeiten tatsächlich zur Kenntnis zu nehmen. Selbst bei einem hohen Spezialisierungsgrad kommt man leicht auf wenigstens eine Dissertation in der Woche. Eine Übersicht zu einem größeren fachlichen Zusammenhang eines Rechtsgebietes ist damit ebenso wenig gegeben wie die Kenntnis relevanter Passagen in Arbeiten, die auf den ersten Blick wenig einschlägig erscheinen. Und dabei ist nur von Dis-

39 Dass Zeit- und Entscheidungsdruck zu einer Präferenz des elektronischen Formats führt, hat auch Wissenschaftsrat, Perspektiven der Rechtswissenschaft in Deutschland - Situation, Analysen, Empfehlungen, Köln 2012, S. 37 festgestellt. 
sertationen die Rede. Wenn man noch einschlägige Aufsätze und dergleichen hinzurechnet, dürfte eine in der Summe erdrückende Leselast zusammenkommen. ${ }^{40}$ Hier versprechen neue Verfahren des Textmining Abhilfe, die automatisch aus einer großen Menge an Publikationen relevante Inhalte identifizieren und inhaltlich auswerten können. ${ }^{41}$ Mag sich hier auch vieles noch in einem früheren Entwicklungsstadium befinden, so darf man sicher sein, dass entsprechende Technologien der „Maschinenlektüre“ in absehbarer Zeit zur Verfügung stehen werden. Wie auch immer diese Verfahren dann funktionieren, eine Voraussetzung muss in jedem Fall gegeben sein, nämlich der freie Zugang zum Content, um das Textmining erfolgreich durchführen zu können. Hier würden Zugangsbeschränkungen im Ergebnis bedeuten, dass entsprechende Inhalte nicht oder nur sehr nachrangig zur Kenntnis genommen werden. Allein aus Kapazitätsgründen wird die Wissenschafts- bzw. Forschungskommunikation der Zukunft auf Textmining-Verfahren nicht verzichten können. Daraus dürfte sich allein schon um der zuverlässigen Kenntnisnahme willen ein faktischer Zwang zu Open Access auch und gerade für forschungsintensive Texte wie juristische Dissertationen ergeben. ${ }^{42}$

\section{J. Eine freiwillige Angelegenheit?}

Die Frage jedoch, ob Open Access eine freiwillige Entscheidung von Autorinnen und Autoren sein soll, wird gerade bei buchaffinen Disziplinen, zu denen ja auch die Rechtswissenschaft gehört, seit mehreren Jahren leidenschaftlich diskutiert. ${ }^{43}$ Betont wird hier das Recht der Autorin bzw. des Autors an ihrem bzw. seinem Werk und eine damit gegebene Werkherrschaft, die auch die Entscheidung, in wel-

40 Schroeder, Die Entwicklung der juristischen Promotionen in Deutschland, in: JuS 1998, S. 285 rechnet vor, dass ein Zivilrechtler 504 Dissertationen im Jahr zur Kenntnis nehmen müsste. Diese Zahl wird man angesichts gestiegener Promotionszahlen heute sicher nach oben korrigieren müssen.

41 Vgl. Tobschall/Kempe, Der deutsche Legal-Tech-Markt, in: Breidenbach/Glatz (Hrsg.), Rechtshandbuch LegalTech, München 2018, Kap. 1.4, Rn. 8, sowie Krimphove/Niehaus, Maschinelle Entscheidungsfindung: Der Einsatz der Fuzzy-Logik in der Rechtswissenschaft, ebendort, Kap. 9.3, Rn. 15 f.

42 Auf die positiven Effekte von Open Access für die akademische Integrität durch die leichtere Entdeckung von Plagiaten sei an dieser Stelle nur hingewiesen.

43 Besonders meinungsfreudig sind hier die publizistischen Beiträge des Heidelberger Editionsphilologen Roland Reuß, etwa: „Staatsautoritarismus, groß geschrieben - Blauäugigkeit 4.0: Mit seiner Open-Access-Strategie betreibt das Bildungsministerium Forschungspolitik nach Gutsherrenart. Grundrechtsverstöße interessieren nicht. Die Ministerin folgt blind ihren Mitarbeitern “, in: FAZ vom 28. September 2016; „Der Geist gehört dem Staat - Der Open-Access-Wahnsinn geht weiter. Das Land Baden-Württemberg nötigt die Universitäten, ihre wissenschaftlichen Autoren zu knebeln. Konstanz geht mit schlechtem Beispiel voran.“, in: FAZ vom 30. Dezember 2015; „Ein gravierender Angriff auf die Freiheit der Forschung - Open Access als Pflicht“, in: FAZ vom 24. Februar 2014. Zum rechtswissenschaftlichen Diskussionsstand Sandberger, Die Zukunft wissenschaftlichen Publizierens Open Access und Wissenschaftsschranke Anmerkungen zu den Kontroversen über die Weiterentwicklung des Urheberrechts, in: Ordnung der Wissenschaft (OdW) 2017, S. 75-96; Fehling, Verfassungskonforme Ausgestaltung von DFG-Förderbedingungen zur Open-Access-Publikation, in: Ordnung der Wissenschaft (OdW) 2014, S. 113-128, sowie Schmidt, Open Access - hochschulrechtliche Veröffentlichungs- und urheberrechtliche Anbietungspflichten des Hochschulprofessors, Baden-Baden 2016 und Steinhauer, Recht auf Sichtbarkeit - Überlegungen zu Open Access und Wissenschaftsfreiheit, Münster 2010. 
cher Weise und in welchem Medium publiziert wird, mitumfassen soll. ${ }^{44}$ Tatsächlich sprechen gute Gründe für eine solche Publikationsfreiheit von wissenschaftlichen Autorinnen und Autoren. Allein der Umstand, dass in einer gedruckten Publikation im Gegensatz etwa zu einem im Internet frei zugänglichen Inhalt ganz andere Zielgruppen erreicht werden, legt es nahe, dass eben diese Entscheidung für eine bestimmte Zielgruppe genau zu der Freiheit der wissenschaftlichen Kommunikation gehört, die das Grundrecht der Wissenschaftsfreiheit in Art. 5 Abs. 3 GG schützen will. ${ }^{45}$ Gerade bei politisch oder gesellschaftlich kontroversen Themen kann die Entscheidung, einen wissenschaftlichen Beitrag nur innerhalb der engeren wissenschaftlichen Fachwelt zu publizieren anstatt ihn einer womöglich sehr unsachlichen Anprangerung in sozialen Medien auszusetzen, wenn er frei zugänglich im Netz verfügbar ist, sehr gut nachvollzogen werden. ${ }^{46}$ Und dennoch ist diese Sicht nicht konsequent durchzuhalten. Auch eine Buchpublikation ist eine Veröffentlichung und kann als solche jederzeit und von jedermann zum Gegenstand einer öffentlichen Debatte in Online-Foren gemacht werden. Hier wird deutlich, dass die Idee einer souveränen Werkherrschaft den öffentlichen Charakter wissenschaftlicher Diskurse verfehlt. ${ }^{47}$ Es ist eben nicht Sache einzelner Autorinnen und Autoren, den medialen Rahmen einer wissenschaftlichen Diskussion zu bestimmen, ${ }^{48}$ sondern die Entscheidung einer wissenschaftlichen Community, in der sich in eine konkrete Kommunikationspraxis herausbildet, die für jeden, der am Diskurs teilnehmen möchte, eine normative Wirkung entfaltet. Hier steht die im Zusammenhang mit Open Access gerne behauptete Publikationsfreiheit des einzelnen der ebenfalls verfassungsrechtlich geschützten Rezeptionsfreiheit der vielen gegenüber und muss sich in praktischer Konkordanz auch einer persönlich nicht favorisierten Rezeptionssituation öffnen, wenn diese in einer bestimmten Wissenschaft Standard

44 Vgl. Reuß/Rieble (Hrsg.), Autorschaft als Werkherrschaft in digitaler Zeit, Frankfurt am Main 2009.

45 Zur Wissenschaftsfreiheit als Kommunikationsfreiheit vgl. Geiger, Wissenschaftsfreiheit als Problem der politischen Ordnung in der Bundesrepublik Deutschland, in: König (Hrsg.), Wesen und Aufgabe einer katholischen Universität, Düsseldorf 1984, S. 102 f.; Schmidt-Aßmann, Die Wissenschaftsfreiheit nach Art. 5 Abs. 3 GG als Organisationsgrundrecht, in: Becker (u.a.) (Hrsg.) Festschrift für Werner Thieme zum 70. Geburtstag, Köln [u.a.], 1993., S. 698, 702; Trute, Art. „Wissenschaftsfreiheit“, in: Heun (u.a.) (Hrsg.), Evangelisches Staatslexikon, Stuttgart 2006, Sp. 2761.

46 Vgl. zum Problem der anderen Adressaten einer Open Access-Publikation vgl. Steinhauer, Open Access - ein rhetorisches Problem, in: Flachware 4 (2018), S. 38 f.

47 Eindrücklich Kimminich, Das Veröffentlichungsrecht des Wissenschaftlers, in: Wissenschaftsrecht 18 (1985), S. 116 f.: „So ist die Wissenschaft ihrem Wesen nach ein Gespräch, das über alle räumlichen und zeitlichen Grenzen hinweg geführt wird: ein unbegrenztes, grenzenloses Gespräch.“ In dem Beitrag von Kimminich wird übrigens die Frage, ob künftig eher elektronisch publiziert wird, noch ausdrücklich ausgeklammert.

48 So aber Rieble, Autorenfreiheit und Publikationszwang, in: Reuß/Rieble (Hrsg.), Autorschaft als Werkherrschaft im digitalen Zeitalter, Frankfurt am Main 2009, S. 61. 
geworden ist. ${ }^{49}$ Ohne auf die verfassungsdogmatischen Details eingehen zu können, sei ein Gedanke von Dieter Grimm zum Grundrecht der Wissenschaftsfreiheit angeführt. Nach Grimm darf die Wissenschaftsfreiheit nicht gegen die wissenschaftliche Gemeinschaft individualistisch in Stellung gebracht werden. Als Beispiel nennt er den Verzicht der Veröffentlichung von Forschungsergebnissen, die aus Kooperationen mit privatwirtschaftlichen Unternehmen gewonnen wurden. Dies sei ein funktionswidriger Grundrechtsgebrauch, der um der Wissenschaftsfreiheit willen nicht hinzunehmen sei. ${ }^{50}$ Dieser Gedanke lässt sich auch auf die Diskussion um eine Pflicht zu Open Access übertragen.

Für die Gesamtheit des rechtswissenschaftlichen Schrifttums kann man derzeit vermutlich nicht von einer wissenschaftsimmanenten Notwendigkeit von Open Access reden. Die Rechtsquellen selbst sind wegen $\$ 5$ UrhG frei zugänglich und dank großer Fachdatenbanken und einer durch diese auch geförderten Konzentration auf eine relativ überschaubare Anzahl von Kommentaren und Zeitschriften, dürfte der juristische Diskursraum wenig Informationsengpässe enthalten. Das gilt, wie eingangs gezeigt wurde, aber nicht für die juristischen Dissertationen, die kaum verbreitet, wenig rezipiert und daher faktisch unsichtbar sind.

Diese mangelnde Sichtbarkeit entwertet einen wenigstens zahlenmäßig erheblichen Teil der rechtswissenschaftlichen Forschung in Deutschland. ${ }^{51}$ Aus wissenschaftsimmanenten Gründen ist hier Abhilfe geboten. Eine strikte Rechtspflicht zu Open Access wäre die richtige Antwort auf diesen mit Blick auf die Effizienz und Nachhaltigkeit von Forschung unhaltbaren Zustand. ${ }^{52}$ Wenn dagegen eine Verletzung etwa des geistigen Eigentums in Form urheberrechtlicher Verwertungsrechte ins Feld geführt wird, so darf daran erinnert werden, dass eine für Autorinnen und Autoren weitgehend kostenfreie Open Access-Publikation einen wesentlichen geringeren Eingriff in das Grundrecht aus Art. 14 GG darstellt als die frühere Ablieferungspflicht einer dreistelligen Anzahl von Tauschexemplaren auf Kosten der Promovierenden. Wurde diese nicht unerhebliche Belastung des Eigentumsgrundrechts trotz einiger Kritik um der angemessenen Verbreitung der Dissertation als

49 Vgl. Steinhauer, Das Recht auf Sichtbarkeit - Überlegungen zu Open Access und Wissenschaftsfreiheit, S. 71 f.: „Der Freiheit des Einzelnen, zu publizieren, wo er will, steht die Freiheit der Vielen gegenüber, die für ihre wissenschaftliche Arbeit einen einfachen Zugang zu wissenschaftlichen Publikationen benötigen".

50 Vgl. Grimm, Wissenschaftsfreiheit vor neuen Grenzen, Göttingen 2007, S. 28 f.

51 Nach Schroeder, Die Entwicklung der juristischen Promotionen in Deutschland, in: JuS 1998, S. 281 sind Dissertationen ein wesentlicher Teil der Forschung an den juristischen Fakultäten und bilden einen „großen Teil des juristischen Schrifttums“.

52 Bemerkenswert ist in diesem Zusammenhang die Äußerung des eher verlagsfreundlich gesinnten Abgeordneten Ansgar Heveling (CDU) im Rahmen einer Plenardebatte zum Thema Open Access im Wissenschaftsbereich am 29. September 2011 im Deutschen Bundestag, der „Auflagen in den Promotionsordnungen“ zur Förderung von Open Access für „problemlos“ möglich hält, vgl. PlPr. 17/130, S. 15.370 (D). 
im Ergebnis rechtmäßig angesehen, ${ }^{53}$ so dürfte demgegenüber die Eingriffsintensität einer Open Access-Pflicht kaum die Schwelle der Grundrechtsbelästigung überschreiten. Die starke Überhöhung der Bedeutung des geistigen Eigentums in der aktuellen Debatte um Open Access ist im Vergleich zu der finanziellen Belastung durch die Ablieferungspflicht vieler Tauschexemplare in der älteren Promotionspraxis kaum nachvollziehbar.

Allerdings soll nicht übersehen werden, dass eine flächendeckende Etablierung von Open Access bei den rechtswissenschaftlichen Promotionen jenseits der dann erreichten bloßen Zugänglichkeit dieser Arbeiten auch weitere Fragen aufwirft. Hier geht es zum einen um die traditionelle Form der Dissertation als gedrucktes Buch, die bei einer digitalen Publikation infrage gestellt wird, und um die Rolle der rechtswissenschaftlichen Verlage.

\section{K. Ein Wort zum Buch!}

So unbestritten vorteilhaft und praktisch vor allem für die punktuelle Rezeption es ist, wenn eine Publikation in digitaler Form frei zugänglich über das Internet zur Verfügung steht, für die intensive Lektüre gerade eines längeren Textes bietet die traditionelle Buchform immer noch große Vorteile. Es wäre sicher ein Verlust, wenn als Folge der Etablierung von Open Access bei den rechtswissenschaftlichen Dissertationen keine gedruckten Arbeiten mehr verfügbar wären. Ein nicht geringer Teil der in der Praxis zu beobachtenden Skepsis gegenüber Open Access und Online-Dissertationen hat in der medialen Präferenz für das Buch seinen Ursprung. Dies gilt nicht nur für die Seite der Leserinnen und Leser solcher Arbeiten, sondern in hohem Maße auch für die Autorinnen und Autoren selbst. Sie wollen nicht darauf verzichten, als im Wortsinn greifbares Ergebnis ihrer jahrelangen Mühe am Ende ein richtiges Buch in der Hand zu halten.

Open Access und die Publikation eines Buches müssen sich aber nicht ausschließen. Im Wege des Print on demand können parallel zur frei zugänglichen OnlineFassung einer Arbeit qualitativ hochwertige Leseexemplare zu wirtschaftlichen Bedingungen hergestellt und angeboten werden. ${ }^{54} \mathrm{Da}$ das Buch und die Online-Fassung auf unterschiedliche Nutzungsszenarien zielen, können sich beide Versionen sogar gegenseitig fördern. Angesichts der äußerst geringen Verbreitung gedruckter Dissertationen dürfte die Zahl derjenigen, die durch eine im Internet frei zugängliche Arbeit auf einen bestimmten Titel aufmerksam werden und ihn für die intensi-

53 Dazu in der älteren Literatur Schnieders, Druckzwang für Dissertationen und Dissertationentausch : eine Erörterung von Grundfragen anhand der historischen Entwicklung in Deutschland und der gegenwärtigen Situation im Land Berlin, Köln 1972, S. 83-85; Walter, Dissertations-Druckzwang und Grundgesetz, in: DVBl. 1972, S. $311 \mathrm{f}$.

54 Dazu Steinhauer, Hybrides Publizieren als Marketing-Mix - Erfolgsmodell zur Verbreitung von Hochschulschriften und wissenschaftlichen Monografien, in: BuB Forum Bibliothek und Information 59 (2007), S. 280-283. 
ve Lektüre kaufen, höher sein als die Zahl derjenigen, die wegen der freien Verfügbarkeit von einem Kauf absehen. Das kann allerdings nur funktionieren, wenn die Preise für gedruckte Dissertationen moderat sind. Für den Privatkäufer dürfte hier bei $30 €$ die Grenze liegen. Angemerkt sei, dass diese Art der Verbreitung juristischer Dissertationen wegen der leichteren Sichtbarkeit und Auffindbarkeit deren Rezeption sicher steigern wird, auch und gerade bei der privaten intensiven Lektüre. Natürlich darf man hier keine überzogenen Erwartungen haben, aber die Steigerung echter Leserinnen und Leser von vielleicht 5 bis 10 auf mehr als 30 wäre schon ein enormer Erfolg. Es darf nicht vergessen werden, dass juristische Dissertationen nun einmal hochspezielle Nischenliteratur sind.

\section{Haben Verlage noch eine Zukunft?}

Auch wenn Open Access und die Existenz gedruckter Bücher sich nicht ausschließen, so ist fraglich, ob dies auch für die Rolle der Verlage bei der Publikation und Verbreitung von rechtswissenschaftlichen Dissertationen gilt. Im Vergleich zur überkommenen Tauschdissertation, die praktisch nur in Universitätsbibliotheken verfügbar ist, sollte es die besondere Aufgabe von Verlagen sein, für eine bessere Verbreitung zu sorgen. Wegen der Verfügbarkeit im Buchhandel sind Dissertationen ja nicht mehr nur in wissenschaftlichen Bibliotheken zu finden, sondern zielen auch auf die Justiz, die Anwaltschaft und die interessierte Öffentlichkeit als Abnehmer. Wir hatten jedoch gesehen, dass Verlagsdissertationen jedenfalls im Diskursraum der Juristischen Fakultäten nur unzureichend verbreitet sind. Es ist kaum anzunehmen, dass es in der Justiz oder der Anwaltschaft grundlegend anders ist. Im Vergleich zu Open Access können Verlage sicher nicht darauf verweisen, dass sie juristische Dissertationen besonders erfolgreich verbreitet haben. Allerdings erbringen Verlage etwa durch die Pflege von Schriftenreihen oder besondere Qualitätsanforderungen eine Orientierungsleistung, die reine Open Access Plattformen in dieser Form (noch?) nicht leisten können. Diese Plattformen verbreiten Inhalte durch Metadaten entlang einer meist bibliothekarisch organisierten Informationsinfrastruktur. Hier können Verlage weiterhin eine zusätzliche Sichtbarkeit durch besondere Werbemaßnahmen oder eine fachlich-qualitative Selektion organisieren. Sie können die Arbeiten zudem professioneller, als institutionelle oder fachliche Repositorien dies vermögen, digital aufbereiten und auf eigenen Plattformen durch Verlinkungen und andere Mehrwerte angereichert anbieten. Überdies können Verlage gut gestaltete Buchausgaben wie bisher weiterhin exklusiv vermarkten. Allerdings darf nicht übersehen werden, dass das auf mitunter erheblichen Druckkostenzuschüssen aufgebaute Geschäftsmodell reiner Dissertationsverlage, die neben der bloßen Publikation keinen besonderen Mehrwert anbieten, durch eine umfassende Open Access-Pflicht für rechtswissenschaftliche Dissertationen kaum Bestand haben wird. 
Generell kann die Sorge um die Verlage, die juristische Dissertationen anbieten, in der Frage für oder gegen Open Access keine große Rolle spielen. Bei dieser Textsorte publizieren Verlage zunächst nur, was ihnen angeboten wird. Zudem werden Form und Inhalt von einem Prüfungsamt bzw. Prüfungsausschuss vorgegeben. Dissertationen eröffnen anders als Handbücher, Lehrbücher oder Kommentare nur wenig verlegerischen Gestaltungspielraum. Verlage haben hier tatsächlich nur die Funktion, die von der Wissenschaft her geforderte Verbreitung zu organisieren. Wenn sie damit nicht mehr erfolgreich sind, wofür derzeit viel spricht, werden sie überflüssig. ${ }^{55}$ Aus der rein dienenden Funktion der Verlage bei der Publikation von Dissertationen folgt jedenfalls, dass sie ihre Arbeit an den Bedürfnissen der Wissenschaft ausrichten und daraus ihr Geschäftsmodell entwickeln müssen, ${ }^{56}$ wenn sie denn noch eine Zukunft haben wollen.

\section{Zusammenfassung und Ausblick}

Die Verbreitung juristischer Dissertationen ist derzeit äußerst mangelhaft, obwohl sie als aktuelle Forschungsleistungen der wissenschaftlichen Öffentlichkeit in angemessener Weise zugänglich sein sollen. Eine umfassende Open Access-Pflicht für Dissertationen könnte hier Abhilfe schaffen, zumal angesichts der großen Anzahl an neuen Arbeiten, die jedes Jahr erscheinen, eine physische Sammlung dieser Werke in Bibliotheken selbst bei ausreichendem Erwerbungsetat kaum organisiert werden kann. Zwar bieten auch Verlage mittlerweile digitale Sammlungen zur Lizenzierung an, doch erschweren Zugangsbarrieren die interdisziplinäre Rezeption und die Sichtbarkeit in der juristischen Praxis.

Generell sollte die Diskussion nach dem angemessenen Weg, eine juristische Dissertation zu publizieren, von Fragen der Sichtbarkeit und der Rezeption bestimmt sein. Die oft zu hörende Ansicht, dass es allein darauf ankomme, in einem „guten Verlag“ oder in einer „renommierten Reihe“ zu publizieren, verfehlt angesichts der tatsächlichen Verbreitung von Verlagsdissertationen eigentlich das Thema, es sei denn, man wertet eine Publikation allein als Status-Symbol. ${ }^{57}$ Das aber wäre eine wissenschaftsfremde Sicht, die die Entwicklung einer sachgerechten Publikationspraxis rechtswissenschaftlicher Dissertationen nicht bestimmten sollte. Auch die von Verlagen immer wieder hervorgehobene Qualitätskontrolle durch Schriftenreihen scheint sich auf die tatsächliche Verbreitung einer Arbeit in den Bibliotheken

$55 \mathrm{Zu}$ Perspektiven der Wissenschaftsmonographie unter den Bedingungen von Open Access vgl. Näder, Open Access - Wissenschaftliche Verwertung im Zeitalter von Digitalität und Internet, Dresden 2010, S. $90 \mathrm{f}$.

56 Im Ergebnis auch Link, Open Access im Wissenschaftsbereich, Frankfurt am Main 2013, S. 189.

57 Die Sicht, Dissertationen nicht als wissenschaftlichen Beitrag, sondern als bloße Selbstdarstellung zu werten, ist nicht neu, vgl. Fabian, Buch, Bibliothek und geisteswissenschaftliche Forschung, Göttingen 1983, S. 248-253. Tatsächlich nimmt die Frage der optimalen Selbstvermarktung durch den Veröffentlichungsort bei Brandt, Dr. jur. - Wege zu einer erfolgreichen Promotion, Baden-Baden 2018, Rn. 292 einen erheblichen Raum ein. 
kaum auszuwirken. Bei einer Prädikatsquote von rund $70 \%$ bei juristischen Dissertationen ist die Selektionswirkung durch die überdies nur wenigen Verlage, die juristische Dissertationen verlegen, eher gering. ${ }^{58}$ Angemerkt sei, dass die prominenten Plagiatsfälle der vergangenen Jahre meist in Schriftenreihen renommierter Verlage erschienen sind. Am Ende sind es die Juristischen Fakultäten, die die eigentliche Qualitätskontrolle leisten oder nicht.

Die vorstehenden Überlegungen dürften gezeigt haben, dass es zu Open Access bei juristischen Dissertationen eigentlich keine sinnvolle Alternative gibt. Diskutiert werden muss aber, wie man diese Openness richtig organisiert und welche sinnvollen und begleitenden Dienstleistungen die juristischen Verlage hier in Zukunft anbieten können. Es geht am Ende nicht darum, das freie Internet gegen die Verlage auszuspielen, ${ }^{59}$ sondern gemeinsam das Problem einer unzureichenden Sichtbarkeit und Verbreitung aktueller rechtswissenschaftlicher Forschung in Gestalt der Dissertationen anzugehen und einer für die Bedürfnisse der Praxis sinnvollen und zukunftsfähigen Lösung zuzuführen. ${ }^{60}$

58 Die Zahl stammt aus der Datenbank des Deutschen Zentrums für Hochschul- und Wissenschaftsforschung, zugänglich unter: http://www.forschungsinfo.de/promotionsnoten/, und bezieht sich auf die Jahre 2015 bis 2017.

59 Vgl. Hoeren, Elektronische Medien - Aktuelle Entwicklungen und Perspektiven, in: Willoweit (Hrsg.), Rechtswissenschaft und Rechtsliteratur im 20. Jahrhundert, München 2007, S. 1185 f. Hier ist zu bedenken, dass das Internet zwar die Zugänglichkeit zu Inhalten gewährleisten kann, aber damit zugleich nicht automatisch auch Sichtbarkeit gegeben ist. Hier geht es um gute Meta-Daten und Informationsinfrastrukturen. Hier können auch Verlage einen Beitrag zu mehr Sichtbarkeit und einer besseren Auffindbarkeit leisten.

60 So auch Wildgans, Zuckerbrot oder Peitsche? - Ein Plädoyer für Open Access im juristischen Publikationswesen, in: ZUM 2019, S. 28. 